\title{
Rituximab in multiple sclerosis
}

\section{A retrospective observational study on safety and efficacy OPEN}

Jonatan Salzer, MD, PhD

Rasmus Svenningsson

Peter Alping

Lenka Novakova, MD

Anna Björck, MD

Katharina Fink, MD

Protik Islam-Jakobsson,

MD

Clas Malmeström, MD,

$\mathrm{PhD}$

Markus Axelsson, MD,

$\mathrm{PhD}$

Mattias Vågberg, MD

Peter Sundström, MD,

$\mathrm{PhD}$

Jan Lycke, MD, PhD

Fredrik Piehl, MD, PhD

Anders Svenningsson,

$\mathrm{MD}, \mathrm{PhD}$

Correspondence to

Dr. Salzer:

jonatan.salzer@umu.se

Editorial, page 2070

Supplemental data at Neurology.org

\section{ABSTRACT}

Objective: To investigate the safety and efficacy of rituximab in multiple sclerosis (MS).

Methods: In this retrospective uncontrolled observational multicenter study, off-label rituximabtreated patients with MS were identified through the Swedish MS register. Outcome data were collected from the MS register and medical charts. Adverse events (AEs) grades 2-5 according to the Common Terminology Criteria for Adverse Events were recorded.

Results: A total of 822 rituximab-treated patients with MS were identified: 557 relapsingremitting MS (RRMS), 198 secondary progressive MS (SPMS), and 67 primary progressive MS (PPMS). At baseline, 26.2\% had contrast-enhancing lesions (CELs). Patients were treated with 500 or 1,000 mg rituximab IV every 6-12 months, during a mean 21.8 (SD 14.3) months. During treatment, the annualized relapse rates were 0.044 (RRMS), 0.038 (SPMS), and 0.015 (PPMS), and $4.6 \%$ of patients displayed CELs. Median Expanded Disability Status Scale remained unchanged in RRMS $(p=0.42)$ and increased by 0.5 and 1.0 in SPMS and PPMS, respectively ( $p=0.10$ and 0.25 ). Infusion-related AEs occurred during $7.8 \%$ of infusions and most were mild. A total of 89 AEs grades $\geq 2$ (of which 76 infections) were recorded in 72 patients. No case of progressive multifocal leukoencephalopathy was detected.

Conclusions: This is the largest cohort of patients with MS treated with rituximab reported so far. The safety, clinical, and MRI findings in this heterogeneous real-world cohort treated with different doses of rituximab were similar to those reported in previous randomized controlled trials on B-cell depletion therapy in MS.

Classification of evidence: This study provides Class IV evidence that for patients with MS, rituximab is safe and effective. Neurology ${ }^{\circledR} 2016 ; 87: 2074-2081$

\section{GLOSSARY}

$\mathbf{A E}=$ adverse event; $\mathbf{A R R}=$ annualized relapse rate; $\mathbf{B P F}=$ brain parenchymal fraction; $\mathbf{C E L}=$ contrast-enhancing lesion; CTCAE = Common Terminology Criteria for Adverse Events; DMD = disease modulatory drug; EDSS = Expanded Disability Status Scale; HACA = human antichimeric antibody; HERMES = Helping to Evaluate Rituxan in Relapsing-Remitting Multiple Sclerosis; IgG = immunoglobulin G; JCV = JC virus; $\mathbf{M S}=$ multiple sclerosis; OLYMPUS = A Study to Evaluate the Safety and Efficacy of Rituximab in Adults With Primary Progressive Multiple Sclerosis; PML = progressive multifocal leukoencephalopathy; PPMS = primary progressive multiple sclerosis; $\mathbf{R C T}$ = randomized controlled trial; RRMS = relapsingremitting multiple sclerosis.

Rituximab (Mabthera; Roche, Basel, Switzerland), a chimeric monoclonal B-cell-depleting antiCD20 antibody, has shown beneficial effects in 2 randomized placebo-controlled phase 2 trials (RCTs): the Helping to Evaluate Rituxan in Relapsing-Remitting Multiple Sclerosis (HERMES) trial for relapsing-remitting multiple sclerosis (RRMS) and A Study to Evaluate the Safety and Efficacy of Rituximab in Adults With Primary Progressive Multiple Sclerosis (OLYMPUS) trial for primary progressive multiple sclerosis (PPMS). ${ }^{1,2}$ The notion of a positive effect of antiCD20 antibody treatment in RRMS is supported by 2 recent RCTs with 2 new antibodies:

From the Department of Pharmacology and Clinical Neuroscience (J.S., R.S., P.I.-J., M.V., P.S., A.S.), Umeå University; Departments of Clinical Neuroscience (R.S., P.A., A.B., K.F., F.P.) and Clinical Sciences, Danderyd Hospital (A.S.), Karolinska Institutet, Stockholm; and Department of Clinical Neuroscience (L.N., C.M., M.A., J.L.), Institute of Neuroscience and Physiology, Sahlgrenska Academy, University of Gothenburg, Sweden.

Go to Neurology.org for full disclosures. Funding information and disclosures deemed relevant by the authors, if any, are provided at the end of the article. The Article Processing Charge was paid by the authors.

This is an open access article distributed under the terms of the Creative Commons Attribution-NonCommercial-NoDerivatives License 4.0 (CC BY-NC-ND), which permits downloading and sharing the work provided it is properly cited. The work cannot be changed in any way or used commercially. 
ocrelizumab (humanized) and ofatumumab (human). ${ }^{3,4}$ Three large, unpublished RCTs with ocrelizumab in RRMS (A Study of Ocrelizumab in Comparison with Interferon Beta 1a [Rebif] in Patients with Relapsing Multiple Sclerosis [OPERA] I and II) and PPMS (A Study of Ocrelizumab in Patients with Primary Progressive Multiple Sclerosis [ORATORIO]) were presented at the European Conference for Treatment and Research in MS 2015 (ECTRIMS). ${ }^{5}$ In these studies, ocrelizumab showed significant benefit over interferon$\beta$-1a for RRMS and over placebo for PPMS. Furthermore, we recently provided evidence that rituximab is superior to fingolimod regarding disease reactivation in patients switching from natalizumab due to positive JC virus (JCV) serology. ${ }^{6}$

Available data from rituximab in rheumatoid arthritis indicate a high tolerability and low risks for serious opportunistic infections or secondary malignancies. ${ }^{7}$ Progressive multifocal leukoencephalopathy (PML) has been reported in rituximab-treated patients, but usually following other immunosuppressive

Figure 1 Flowchart of case ascertainment

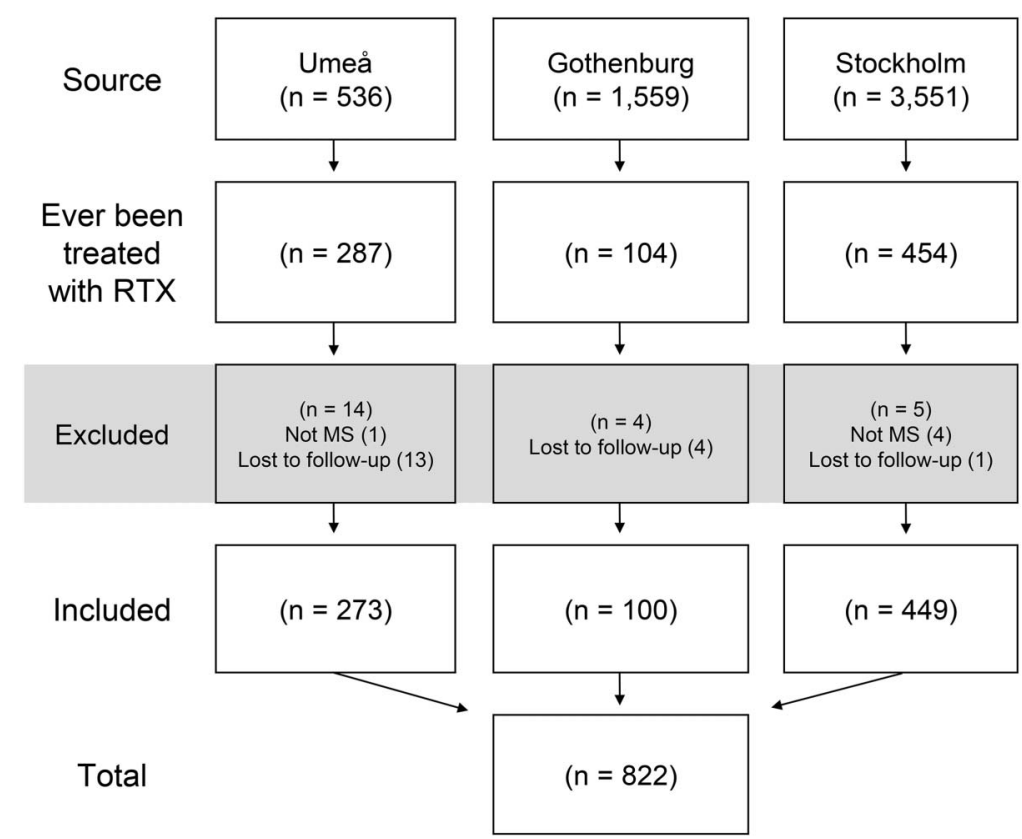

This flowchart depicts how the 822 rituximab (RTX)-treated patients with multiple sclerosis (MS) were identified. The source population was all MS cases registered in the Swedish MS register at the 3 participating MS centers (Umeå University Hospital, Umeå, until April 12, 2015; Karolinska University Hospital, Stockholm, until February 24, 2015; and Sahlgrenska University Hospital, Gothenburg, until April 18, 2015). We excluded patients lost to followup and patients treated with RTX for reasons other than MS (e.g., rheumatoid arthritis, systemic lupus erythematosus, and neuromyelitis optica). treatments in the setting of B-cell lymphoma and rarely in rheumatic diseases. ${ }^{8,9}$ To our knowledge, no case of PML has been reported among rituximab-treated patients with multiple sclerosis (MS).

Since the publication of the HERMES ${ }^{1}$ and OLYMPUS $^{2}$ trials, rituximab has been increasingly used off-label to treat MS in Sweden, in progressive MS with signs of disease activity, and in JCV-positive RRMS with active disease course.

METHODS The primary aim of this retrospective study performed at specialized MS centers at 3 university hospitals in Sweden was to investigate the safety of rituximab in MS (level of evidence IV). The secondary aim was to report the efficacy of rituximab in MS on clinical and MRI measures (level of evidence IV).

Standard protocol approvals, registrations, and patient consents. The study was approved by the local ethics committees in Umeå (2013/445-31) and Stockholm (2009/2107-31/2). Formal patient consent was waived by the ethics committees.

Study population. The source population was all patients with MS ever treated with rituximab recorded in the Swedish MS register launched in 2001 (neuroreg.se) ${ }^{10}$ at the Umeå (until April 12, 2015), Sahlgrenska (Gothenburg, until April 18, 2015), and Karolinska (Stockholm, until February 24, 2015) University Hospitals. Patients treated with rituximab for other concomitant conditions or with no follow-up data available were excluded (figure 1). Medical charts were reviewed according to a prespecified data collection protocol. Time on treatment was defined as time from first rituximab infusion until data censure. For those who discontinued treatment, data collection was extended until 1 year after the last rituximab infusion or time for data censure, whichever came first.

Treatment and follow-up monitoring. Patients were usually treated with single infusions of 500 or 1,000 mg rituximab IV every 6-12 months, in some cases after an initial higher dose treatment course (1,000-2,000 mg subdivided into 2 infusions given within 1 month). Clinical examinations and cerebral 1.5 or 3T MRI were performed routinely every 6-12 months or as clinically indicated. Blood samples for safety and B-cell monitoring were drawn immediately before rituximab infusions. B-cell levels were not used to guide treatment decisions.

Outcome data collection. Clinical and MRI data were retrieved from the Swedish MS register and medical charts. The baseline MRI was defined as the most recent MRI before rituximab treatment initiation. We recorded the presence and numbers of contrastenhancing lesions (CELs) on all MRIs. The brain parenchymal fraction (BPF) is estimated in clinical routine since 2009 at Umeå University Hospital, and was recorded when available. The BPF was calculated using the SyMap method. ${ }^{11}$ Postprocessing was performed using SyMRI BrainStudio version 7.0 (SyntheticMR AB, Linköping, Sweden) with minor manual adjustments to the automated brain segmentation. Patients who discontinued rituximab were considered treated until 1 year after discontinuation. The dates of the most recent relapse before rituximab and all relapses on treatment were recorded. The Expanded Disability Status Scale (EDSS) scores prior to rituximab and the latest EDSS on treatment were recorded. The immunoglobulin $G(\operatorname{IgG}, g / \mathrm{L})$ levels and the number of B cells, expressed as the percentages of CD19-positive cells among the 
CD45-positive cell population (lymphocytes) for Umeå and Gothenburg, and the absolute numbers $\left(10^{6} / \mathrm{mL}\right)$ of CD19positive cells for the Stockholm, on flow cytometry, were recorded when available. The lower normal reference value for IgG levels was $6.2 \mathrm{~g} / \mathrm{L}$, and for B-cell levels $6 \%$ or $0.09 \times 10^{6} / \mathrm{mL}$, respectively. JCV serostatus and titers at baseline were recorded when available.

Adverse events (AEs) occurring during rituximab treatment were registered from medical records covering all medical subspecialties at the hospitals, and graded according to the Common Terminology Criteria for Adverse Events (CTCAE) v 4.03, June 2010. The following AEs were recorded: deaths, malignancies, autoimmune disorders, and infections. We did not record grade $1 \mathrm{AEs}$ or uncomplicated lower urinary or upper respiratory tract infections, since we expected the sensitivity to be low. For the same reason, unspecific symptomatic diagnoses were also excluded. Herpes and herpes zoster infections requiring antiviral treatment were recorded as these may indicate compromised cellular immunity. ${ }^{12}$ Infusion-related AEs (all CTCAE grades) were actively interrogated and recorded in medical charts by the MS nurses at each infusion.

Statistical analyses. SPSS version 23 was used for statistical analyses. Normally distributed variables were presented as mean (SD), parametric variables as median (range). The independent samples $t$ test or 1-way analysis of variance were used to compare means, the Mann-Whitney $U$ test to compare medians. The Pearson $\chi^{2}$ test was used to compare proportions and Fisher exact test if one category had less than 5 observations. The numbers of MRIs with CELs per years of treatment, the annualized relapse rates (ARRs), and the proportions of infusions with infusion reactions were compared using the Upton N-1 $\chi^{2}$ in Win-pepi software version 2.72. We calculated the atrophy rate as the change in BPF $(\triangle \mathrm{BPF})$ divided by years elapsed between each MRI. For patients who had $\geq 2 \Delta$ BPF estimations, we calculated the mean atrophy rate. The $\alpha$ was set at $<0.05$.

RESULTS A total of 822 patients with MS (557 RRMS, 198 SPMS, 67 PPMS) ever treated with rituximab fulfilling the inclusion criteria were identified (figure 1). Of the 557 RRMS cases, $114^{6}$ and $7^{13}$ patients, respectively, were included in 2 other recently published studies. Baseline characteristics are presented in table 1. One-fifth had received rituximab as their first disease modulatory drug (DMD), and the remainder switched from other DMDs, most commonly natalizumab and interferon- $\beta$ (table 1). Median (range) washout periods for first- and second-line therapies were $0.48(0-176.9)$ and $1.22(0-65.2)$ months, respectively. Patients were treated with rituximab during a total of 1,490 and followed during 1,580 patient-years. The mean (SD) treatment duration was 21.8 (14.3) months, median 18.4 (range 0-88), and the mean follow-up time was 23.1 (15.3) months. In total, 313 patients were on treatment for $>24$ months. The median rituximab dose was $1,000 \mathrm{mg}(100-1,000)$ per infusion (table 1). One-third had received 2,000 mg during the first treatment course $(1,000+1,000 \mathrm{mg}$ given within 1 month, table 1). These patients were more likely to have had CELs on their baseline MRI (32.5 vs $22.9 \%, p=0.003$ ), making further analyses based on first treatment course dose difficult.
The mean B-cell levels decreased and remained low over the observed time period (figure 2). IgG levels decreased only slightly on the aggregate level, but 3\% (25 cases) had IgG levels below the lower normal reference value at some point during treatment $(\mathrm{n}=1,107$ sampling occasions, figure 2$)$. The JCV serostatus at baseline was known in 342 patients, of which 285 (83\%) were seropositive. The mean absolute JCV index, determined in 198 patients, was 1.96 (1.22).

Clinical efficacy data. A total of 59 relapses occurred on rituximab treatment, which corresponded to the following ARRs: 0.044 for RRMS, 0.038 for SPMS, and 0.015 for PPMS (figure 3). The relapses occurred at a median of 4.7 (0.16-23.9) months after the most recent infusion. The ARRs on rituximab treatment in patients with RRMS differed across previous treatment categories: 0.016 for treatment-naive patients $(n=119)$, 0.033 for patients previously on first-line DMDs (interferons, glatiramer acetate, dimethylfumarate; $\mathrm{n}=$ 180), and 0.067 for patients previously on second-line DMDs (natalizumab, alemtuzumab; $\mathrm{n}=243)(p=$ 0.015) (15 patients not classifiable). The baseline EDSS $(\mathrm{n}=630)$ was assessed mean 1.8 (2.75) months before rituximab initiation, the latest available followup EDSS ( $\mathrm{n}=613) 22.2$ (14.5) months later. During the observation time, the median EDSS remained unchanged in patients with RRMS ( $p=0.42)$, and increased 0.5 and 1.0 for patients with SPMS and PPMS, respectively ( $p=0.10$ and 0.25 ).

MRI efficacy data. Data for 2,208 MRI examinations were retrieved, including the baseline MRIs. Each patient had a median of 2 (1-9) MRIs including baseline. A baseline MRI was performed in $99.5 \%$ (818 out of 822) of patients, and $77.3 \%$ (635 out of 822) of patients had performed at least 1 MRI after rituximab initiation. The mean time between baseline MRI and rituximab initiation was 4.5 (8.4), and the mean interval between the MRI scans during treatment was 10.6 (6.1) months. At baseline, 26.2\% (214 out of 818) of patients had CELs (a total of 636 CELs, mean [SD] 0.8 [2.3] CELs/MRI, table 1). After treatment initiation, $4.6 \%$ (29 out of 635) of patients had CELs (total 75 CELs on 31 MRIs). When counting all 1,390 MRIs performed after treatment initiation, this yielded a ratio of 0.054 CELs/ MRI, or CELs in $2.2 \%$ of MRIs. The CELs appearing during treatment were more common during the first 6 months vs later $(p<0.001)$ (figure 3). Among the 432 patients with RRMS with data on CELs after rituximab initiation, the numbers of CELs/MRI on treatment were 0.16 in naive patients $(n=82), 0.06$ in patients previously on first-line DMDs $(\mathrm{n}=138)$, and 0.23 in patients previously on second-line DMDs $(\mathrm{n}=198)$ during the first 6 months after the first 


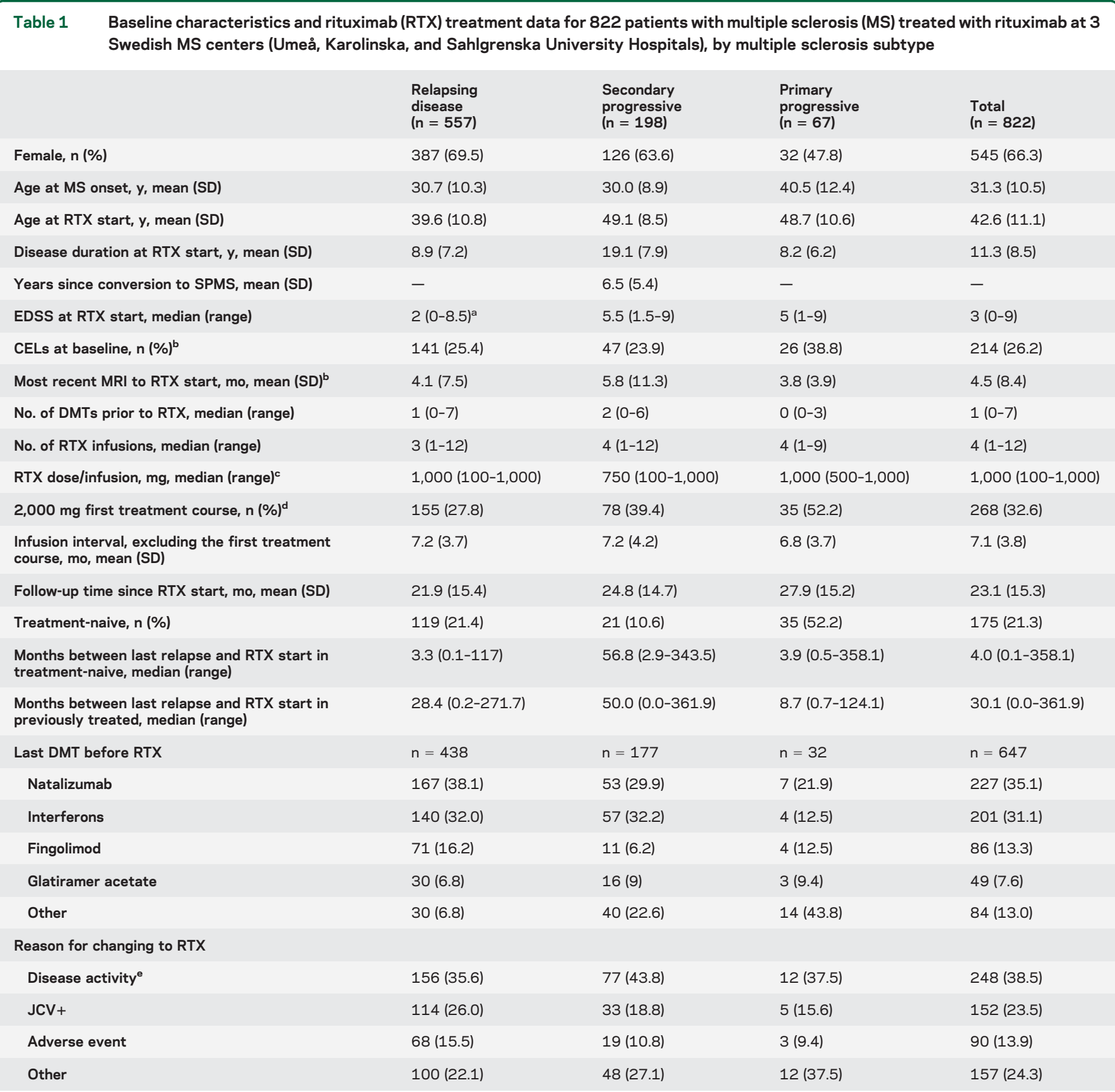

Abbreviations: CEL = contrast-enhancing lesion; DMT = disease-modifying treatment; EDSS = Expanded Disability Status Scale; JCV = JC virus; SPMS = secondary progressive multiple sclerosis.

Data are $\mathrm{n}(\%)$, mean (SD) for continuous variables, or median (range) for nonparametric variables.

a There were 41 patients with relapsing-remitting MS with EDSS scores $\geq 4.5$. Although some of these may have the progressive form of MS, we did not have enough data to reclassify them.

${ }^{b}$ Four cases ( 3 relapsing-remitting MS and 1 SPMS) did not have a baseline MRI.

${ }^{c}$ Values are median (range) of means for each patient.

$\mathrm{d}<1$ month between first and second infusions.

e These patients had experienced a recent relapse, had new or enlarged T2 lesions or CELs on MRI, or were for other reasons deemed to have ongoing disease activity by the treating physician.

rituximab infusion $(p=0.02)$. The corresponding figures during months $6-18$ were $0.01,0.01$, and 0.04 , respectively $(p=0.12)$.

Atrophy rate. The mean annual change in BPF on rituximab treatment was $-0.19 \%(0.95)$. This was assessed in 160 patients at the Umeå University
Hospital who had $\geq 2 \Delta \mathrm{BPF}$ estimations available (726 MRIs).

Rituximab dosing. The 2 most common rituximab protocols in Sweden are 500 and 1,000 mg every 6 months as single maintenance doses after an initial dose that may vary from 500 to $2,000 \mathrm{mg}$, sometimes 
Figure 2 B-cell and immunoglobulin $\mathrm{G}(\lg \mathrm{G})$ levels before and during rituximab treatment in multiple sclerosis cases

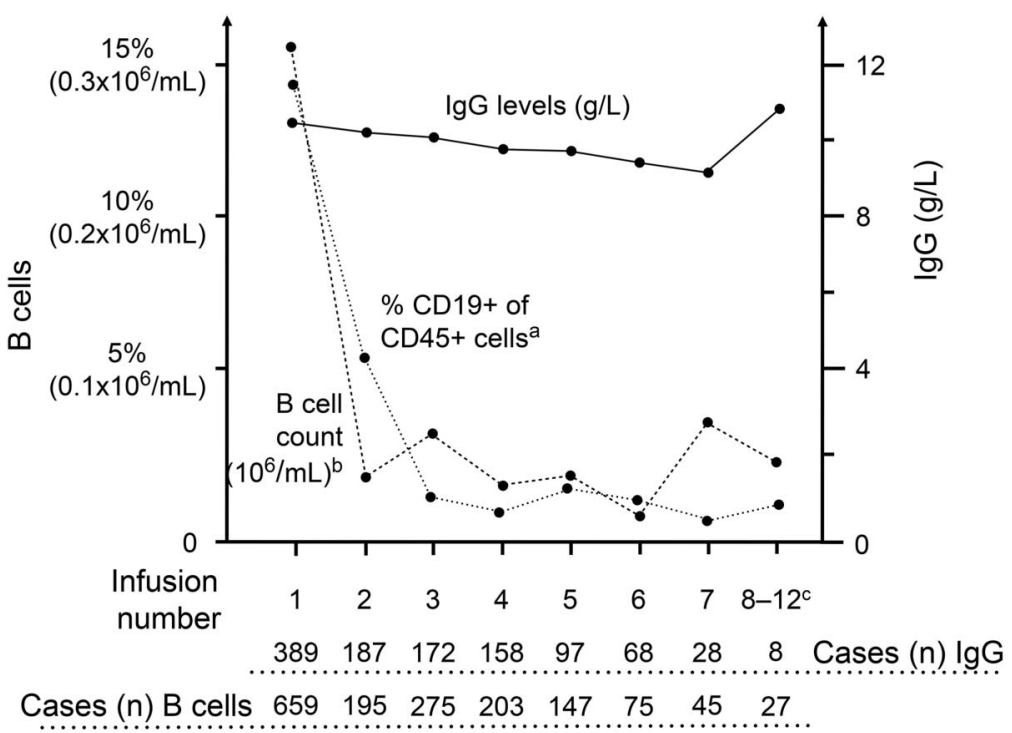

B-cell and IgG levels at samplings immediately before rituximab infusions 1-12. The numbers of cases used to estimate the means and SDs are shown below the figure for IgG (top row) and B cells (lower row). aAt Umeå Hospital and Sahlgrenska University Hospital, the B-cell levels are shown as mean percentage of $\mathrm{CD} 19+$ cells within the $\mathrm{CD} 45+$ cell population. ${ }^{\mathrm{b}} \mathrm{At}$ the Karolinska University Hospital, the B-cell levels are shown as mean absolute numbers $\left(\times 10^{6}\right)$ of CD19+ cells $/ \mathrm{mL}$ blood. cInfusions 8-12 were merged due to few cases.

divided into 2 infusions within 1 month. To compare these 2 treatment regimens, we subdivided the RRMS cohort into those who had received $\leq 750 \mathrm{mg} /$ infusion and those who had received $>750 \mathrm{mg} / \mathrm{infu}-$ sion as maintenance dose. A total of 478 patients with RRMS had received at least 2 infusions and were included in this analysis. The low-dose group $(\mathrm{n}=220)$ had received median $500(100-750)$ and the high-dose group $(\mathrm{n}=258) 1,000(786-2,000) \mathrm{mg}$ rituximab per maintenance treatment course. The majority, $78.2 \%$ in the low-dose group and $82.2 \%$ in the high-dose group, had received 500 and 1,000 mg, respectively, per maintenance infusion. The groups did not differ with regards to most important baseline characteristics, such as proportions with CELs at baseline and previous treatment with natalizumab. However, the lower dose group was slightly older at treatment initiation, 41.1 (10.7) vs 38.6 (10.8) years, $p=0.01$, and had shorter follow-up time, 17.9 (9.6) vs 28.7 (15.7) months, $p<0.01$ (table e-1 at Neurology. org). As CELs were more common on early MRIs, and as the follow-up times for the 2 groups differed, we compared MRI data for the time periods $<6$ and 6-18 months separately. Efficacy data suggested no differences in numbers of CELs/MRI (0.14 vs 0.15 ; $p=0.85)$ during $<6$ months after treatment initiation, or during months $6-18$ (0.02 vs $0.02 ; p=$ 0.67 ), in the 500 vs $1,000 \mathrm{mg}$ groups (table e-1). Also, the ARRs for the entire follow-up period were similar
( 0.040 vs $0.047, p=0.61)$. Non-infusion-related AEs per patient-year of treatment were slightly less common in the 500 vs $1,000 \mathrm{mg}$ groups ( 0.083 vs 0.125$)$ but B-cell and IgG levels did not differ (table e-1).

Adverse events. A total of 89 non-infusion-related AEs grades 2-5 were detected. The most common types of AE were infections $(\mathrm{n}=76)$; this was also true for severe AEs (table 2). Three grade 2 malignancies were detected, and 4 patients died. Causes of death were cardiac arrhythmia, respiratory failure, vascular surgery, and suicide by intoxication, respectively (table e-2).

Infusion reactions (malaise, headache, chills, nausea) occurred during $7.8 \%$ of infusions (234 out of $3,002)$. Such reactions were more common during the first 3 infusions, $10.1 \%$ (213 out of 2,108), compared with subsequent infusions, $2.3 \%$ (21 out of 894) $(p<0.001)$. Infusion-related AE grades were $3(\mathrm{n}=3), 2(\mathrm{n}=72)$, and $1(\mathrm{n}=159)$.

Drug survival. In total, $10.3 \%$ (85 out of 822 ) discontinued rituximab treatment during the study, 43 of these (20 RRMS, 16 SPMS, and 7 PPMS) due to AEs or disease activity. The remainder, 42 patients, stopped treatment due to stable condition, secondary progressive MS, pregnancy, or other reasons (figure e-1). The drug survival (proportion of patients who had not discontinued rituximab due to disease activity or AEs) at data censure was $94.8 \%$ (779 out of 822 , figure e-1).

DISCUSSION We report the largest retrospective observational study so far investigating off-label rituximab treatment in MS. Although data were retrieved from 3 MS centers with different treatment regimens and different selection principles for rituximab treatment, they add important information on safety and tolerability of rituximab in a heterogeneous clinical real-world sample. Due to the design of the study, efficacy data are less reliable but support those achieved in previous RCT studies on rituximab $b^{1,2}$ and other B-cell-depleting therapies, ${ }^{3-5}$ as well as an observational study in a high inflammatory group switching from natalizumab. ${ }^{6}$

In this study, a large proportion of decisions to initiate rituximab treatment were based on MRI. Although we included a relatively high proportion of progressive patients, the formation of new lesions on MRI was high at baseline. Despite this, the observed ARR and MRI disease activity were low during rituximab treatment. Based on the observed ARRs in this study, rituximabtreated patients with RRMS may be expected to experience one relapse every $23 \mathrm{rd}$ year. This is low compared with first-line-agent treated patients with $\mathrm{MS},{ }^{14}$ and even compared with alemtuzumab ${ }^{15}$ - and natalizu$\mathrm{mab}^{16}$-treated patients. Furthermore, the drug survival, reflecting both effectiveness and safety/tolerability, was 


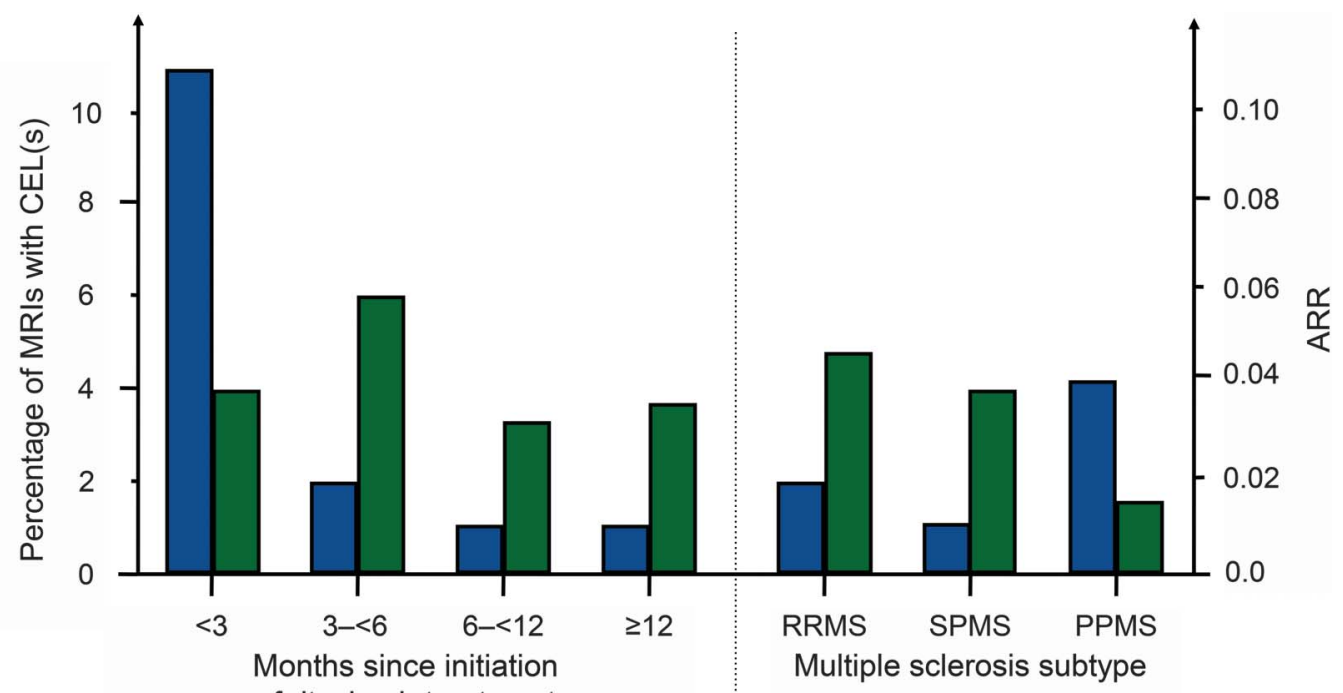

of rituximab treatment

\begin{tabular}{|rcccc|ccc|} 
No. of patients with MS at risk & 822 & 796 & 738 & 602 & 557 & 198 & 67 \\
\hline No. of MRIs & 125 & 176 & 334 & 755 & 1013 & 271 & 106 \\
No. of MRIs with CELs (no. of CELs) & $14(37)$ & $3(3)$ & $4(8)$ & $10(27)$ & $23(56)$ & $3(14)$ & $4(5)$ \\
\hline Percentage of MRIs with CEL(s) & 11 & 2 & 1 & 1 & 2 & 1 & 4 \\
\hline Person-time (years) on rituximab & 202.8 & 192.7 & 338.7 & 755.7 & 981.2 & 372.3 & 136.4 \\
No. of relapses & 8 & 11 & 12 & 28 & 43 & 14 & 2 \\
\hline ARR & 0.039 & 0.057 & 0.035 & 0.037 & 0.044 & 0.038 & 0.015 \\
\hline
\end{tabular}

The percentage of MRIs with contrast-enhancing lesions (CELs, left y-axis, blue bars), as well as the annualized relapse rate (right $y$-axis, green bars), for patients with MS treated with rituximab, by time period after rituximab treatment initiation, or MS subtype ( $x$-axis). The numbers of patients at risk were calculated as the number of patients entering each time period. The person-time variable was calculated as number of years from treatment initiation to data censure, or 12 months after last infusion in case of treatment discontinuation, whichever came first. ARR = annualized relapse rate; PPMS = primary progressive multiple sclerosis; RRMS = relapsing-remitting multiple sclerosis; SPMS = secondary progressive multiple sclerosis.

high compared with earlier reports. ${ }^{17,18}$ Most of the CELs that were detected appeared early (within $\leq 6$ months) after rituximab initiation, suggesting lingering disease activity, which eventually disappeared. However, compared with RCTs, the frequencies of visits and MRI scannings were lower in our study. This probably underestimates numbers of clinical events and transient MRI lesions. Although the different maintenance dose groups, 500 vs $1,000 \mathrm{mg}$ every 6 months, were not identical regarding all baseline parameters, including age, which might have influenced the results, our data suggested no major difference regarding efficacy based on these different dose regimens. Furthermore, slightly fewer AEs per patient-year of treatment occurred in the $500-\mathrm{mg}$ group. Since the emergence of these data, the lower dose treatment protocol has been largely implemented at the 3 sites.

We did not record grade $1 \mathrm{AEs}$ (mild, not treated) for non-infusion-related AEs, as the sensitivity for such events was expected to be low, and it is also likely that some grade $2 \mathrm{AEs}$ (mild, needing intervention) may have been overlooked. However, the expected sensitivity for severe AEs is high since these are likely to be reported by patients or recorded in medical charts. The 4 deaths in this study were not interpreted as rituximab-related (table e-2). We detected no cases of PML, despite the fact that 83.3\% were seropositive among those with known JCV serostatus. However, fewer than half of our patients were on treatment for $>24$ months, and given the low risk of PML even in natalizumab-treated patients during the first 24 months, a longer-term follow-up will be needed to define the PML risk in this patient population. ${ }^{19}$ As for infusion-related AEs, for which the sensitivity is expected to be high as such events are logged by MS nurses, most were mild (grades 1 or 2). In addition, infusion-related AEs were most common during the first 1-3 infusions, indicating that the potential immunogenicity of rituximab is a minor clinical problem. This is of interest in context of human antichimeric antibodies (HACAs). Such antibodies were detected at week 48 in $24.6 \%$ of rituximab-treated patients in the HERMES trial, although no association between the presence of HACAs and AEs or efficacy was seen. ${ }^{1}$ Several authors have speculated about the potential benefits of 
Table 2 Non-infusion-related adverse events (AEs) by severity, type, and frequency for $\mathbf{8 2 2}$ patients with multiple sclerosis treated with rituximab at 3 Swedish multiple sclerosis centers (Umeå, Sahlgrenska, and Karolinska University Hospitals)

\begin{tabular}{|c|c|}
\hline Adverse events & No. (\%) \\
\hline \multicolumn{2}{|l|}{ Any $^{a}$} \\
\hline Grade 2 & $66(8.0)$ \\
\hline Grade 3 & $17(2.1)$ \\
\hline Grade 4 & $2(0.2)$ \\
\hline Grade 5 & $4^{b}(0.5)$ \\
\hline \multicolumn{2}{|l|}{ Severe (CTCAE grades 3 and 4 ) } \\
\hline $\begin{array}{l}\text { Infections (pneumonia, pyelonephritis, sepsis, sinusitis, } \\
\text { appendicitis, enteritis, bronchitis, erysipelas, intestinal } \\
\text { abscess, tubulointerstitial nephritis) }\end{array}$ & $14(1.7)$ \\
\hline Cardiac disorder (acute coronary syndrome) & $1(0.1)$ \\
\hline Respiratory disorder (interstitial pneumonitis) & $1(0.1)$ \\
\hline Nervous system disorder (bilateral facial palsy) & $1(0.1)$ \\
\hline Immune system disorder (rheumatoid arthritis) & $1(0.1)$ \\
\hline Skin disorder (Sweet syndrome) & $1(0.1)$ \\
\hline \multicolumn{2}{|l|}{ Malignancies (all CTCAE grade 2) } \\
\hline Basalioma & $2(0.2)$ \\
\hline Pyoderma gangrenosum & $1(0.1)$ \\
\hline \multicolumn{2}{|l|}{ Most common ( $\geq 4$ cases) infections } \\
\hline Pneumonia & $19(2.3)$ \\
\hline Otitis & $12(1.5$ \\
\hline Sinusitis & $10(1.2$ \\
\hline Herpes & $6(0.7)$ \\
\hline Gastroenteritis & $5(0.6)$ \\
\hline Herpes zoster & $4(0.5)$ \\
\hline Bronchitis & $4(0.5)$ \\
\hline
\end{tabular}

AEs by disease course

Relapsing-remitting MS

68 (12.2)

Secondary progressive MS

$16(8.9)$

Primary progressive MS

Abbreviations: $\mathrm{CTCAE}=$ Common Terminology Criteria for Adverse Events, version 4.03; MS = multiple sclerosis.

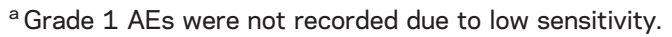

${ }^{b}$ Causes of death in these 4 cases were cardiac arrhythmia, respiratory failure, vascular surgery, and suicide, respectively. More detailed data are available in table e-2.

humanized or human anti-CD20 antibodies regarding lower immunogenicity, ${ }^{3,4}$ but so far no data to corroborate this have been presented.

The mechanisms of action for anti-CD20 treatment in MS are unknown, but may include immune modulation through a reduction in B-cell-dependent antigen presentation, less B-cell-dependent granulocyte macrophage-colony stimulating factor secretion, or lower levels of autoreactive antibodies. ${ }^{20}$ The concordance of efficacy data across different B-cell-depleting agents suggests that the results represent a class effect rather than specific effects mediated

by different binding epitopes or the balance between different effector mechanisms between the different anti-CD20 monoclonal antibodies. ${ }^{1-5}$

Weaknesses of this study apart from those already mentioned include the lack of a control group and the retrospective design, which has inherent methodological issues. These concern data quality, e.g., insufficient documentation in medical charts, and low outcome sensitivity, which prevented grade $1 \mathrm{AE}$ reporting. Also, different methods of reporting B-cell levels prevented complete analyses of this variable on the cohort as a whole, and the low number of patients with cerebral volumetric estimations as well as the lack of a control group for this measure limits the usefulness of atrophy data.

This observational study provides level IV evidence that rituximab is safe and effective for treating MS for up to 2 years. A phase 3 RCT is motivated and may be performed as an investigator-driven effort. This should be given high priority for public funding agencies given the potential patient and societal (low treatment costs) benefits.

\section{AUTHOR CONTRIBUTIONS}

The study was conceived and designed by A.S., J.S., F.P., P.A., and J.L. Data collection was performed by R.S., P.A., L.N., A.B., K.F., P.I.-J., C.M., M.A., A.S., J.S., and F.P. Statistical analyses were performed by J.S. The report, tables, and figures were drafted by J.S., who had full access to all data. All authors provided comments and intellectual input on the tables, figures, analyses, interpretation of data, and manuscript draft. All authors approved the final version for publication.

\section{ACKNOWLEDGMENT}

The authors thank Carl Piehl for primary data collection in the Stockholm cohort and doctors and nurses at all 3 sites for providing clinical data on their patients.

\section{STUDY FUNDING}

No targeted funding reported.

\section{DISCLOSURE}

J. Salzer has received lecture honoraria from BiogenIdec, Teva Pharmaceuticals, and Genzyme/Sanofi, has received travel support from BiogenIdec, and has received research support from Synapsys. R. Svenningsson and P. Alping report no disclosures relevant to the manuscript. L. Novakova has received travel support and/or lecture honoraria from Biogen and Novartis and unconditional research grant from Biogen. A. Björck reports no disclosures relevant to the manuscript. K. Fink has received an unrestricted research grant from Biogen. P. Islam-Jakobsson reports no disclosures relevant to the manuscript. C. Malmeström has received lecture honoraria and had travel expenses partially reimbursed from Biogen, Merck-Serono, and Novartis. He has received a nonconditional research grant from Biogen and served on advisory boards for Biogen, Novartis, and Roche. M. Axelsson has received travel support and/or lecture and writing honoraria from Biogen, Novartis, and Genzyme/SanofiAventis, has served on scientific advisory boards for Biogen, Novartis, and Genzyme/SanofiAventis, and has received unconditional research grants from Biogen. M. Vågberg has received unconditional research grants from BiogenIdec $A B$ and Neuro Sweden, has received lecture honoraria from BiogenIdec $A B$, has received travel grants from BiogenIdec $A B$, Novartis, and Baxter Medical $A B$, has received writing honoraria from Pharma Industry and BestPractice Multiple Sclerosis. P. Sundström has received honoraria from Biogen for serving as a member of a stipend committee. J. Lycke has received travel support and/or lecture honoraria from Biogen, Novartis, Teva, and Genzyme/SanofiAventis, has 
served on scientific advisory boards for Almirall, Teva, Biogen, Novartis, and Genzyme/SanofiAventis, serves on the editorial board of Acta Neurologica Scandinavica, and has received unconditional research grants from Biogen, Novartis, and Teva. F. Piehl has received unrestricted academic research grants from Biogen and Novartis. A. Svenningsson has served on an advisory board for Sanofi-Genzyme and has received travel funding from Biogen Idec. Go to Neurology.org for full disclosures.

Received February 8, 2016. Accepted in final form June 2, 2016.

\section{REFERENCES}

1. Hauser SL, Waubant E, Arnold DL, et al. B-cell depletion with rituximab in relapsing-remitting multiple sclerosis. N Engl J Med 2008;358:676-688.

2. Hawker K, O'Connor P, Freedman MS, et al. Rituximab in patients with primary progressive multiple sclerosis: results of a randomized double-blind placebo-controlled multicenter trial. Ann Neurol 2009;66:460-471.

3. Kappos L, Li D, Calabresi PA, et al. Ocrelizumab in relapsingremitting multiple sclerosis: a phase 2 , randomised, placebocontrolled, multicentre trial. Lancet 2011;378:1779-1787.

4. Sorensen PS, Lisby S, Grove R, et al. Safety and efficacy of ofatumumab in relapsing-remitting multiple sclerosis: a phase 2 study. Neurology 2014;82:573-581.

5. Fyfe I. In the news: ocrelizumab excites ECTRIMS. Nat Rev Neurol 2015;11:667.

6. Alping P, Frisell T, Novakova L, et al. Rituximab versus fingolimod after natalizumab in multiple sclerosis patients. Ann Neurol 2016;79:950-958.

7. van Vollenhoven RF, Emery P, Bingham CO III, et al. Long-term safety of rituximab in rheumatoid arthritis: 9.5year follow-up of the global clinical trial programme with a focus on adverse events of interest in RA patients. Ann Rheum Dis 2013;72:1496-1502.

8. Clifford DB, Ances B, Costello C, et al. Rituximab-associated progressive multifocal leukoencephalopathy in rheumatoid arthritis. Arch Neurol 2011;68:1156-1164.

9. Sikkema T, Schuiling WJ, Hoogendoorn M. Progressive multifocal leukoencephalopathy during treatment with rituximab and CHOP chemotherapy in a patient with a diffuse large B-cell lymphoma. BMJ Case Rep 2013:2013.
10. Hillert J, Stawiarz L. The Swedish MS registry: clinical support tool and scientific resource. Acta Neurol Scand 2015;132:11-19.

11. Vagberg M, Lindqvist T, Ambarki K, et al. Automated determination of brain parenchymal fraction in multiple sclerosis. AJNR Am J Neuroradiol 2013;34: 498-504.

12. Kim JW, Min CK, Mun YC, et al. Varicella-zoster virusspecific cell-mediated immunity and herpes zoster development in multiple myeloma patients receiving bortezomib- or thalidomide-based chemotherapy. J Clin Virol 2015;73: 64-69.

13. Salzer J, Lycke J, Wickstrom R, Naver H, Piehl F, Svenningsson A. Rituximab in paediatric onset multiple sclerosis: a case series. J Neurol 2016;263:322-326.

14. PRISMS Study Group and the University of British Columbia MS/MRI Analysis Group. PRISMS-4: long-term efficacy of interferon-beta-1a in relapsing MS. Neurology 2001;56: $1628-1636$

15. Willis MD, Harding KE, Pickersgill TP, et al. Alemtuzumab for multiple sclerosis: long term follow-up in a multicentre cohort. Mult Scler 2016;22:1215-1223.

16. Butzkueven H, Kappos L, Pellegrini F, et al. Efficacy and safety of natalizumab in multiple sclerosis: interim observational programme results. J Neurol Neurosurg Psychiatry 2014;85:1190-1197.

17. Rotstein DL, Healy BC, Malik MT, Chitnis T, Weiner HL. Evaluation of no evidence of disease activity in a 7year longitudinal multiple sclerosis cohort. JAMA Neurol 2015;72:152-158.

18. Frisell T, Forsberg L, Nordin N, et al. Comparative analysis of first-year fingolimod and natalizumab drug discontinuation among Swedish patients with multiple sclerosis. Mult Scler 2016;22:85-93

19. McGuigan C, Craner M, Guadagno J, et al. Stratification and monitoring of natalizumab-associated progressive multifocal leukoencephalopathy risk: recommendations from an expert group. J Neurol Neurosurg Psychiatry 2016;87: $117-125$

20. Hauser SL. The Charcot Lecture: beating MS: a story of B cells, with twists and turns. Mult Scler 2015;21:8-21.

\section{Help Get the New Congress to Work for Neurology}

The 2016 election may bring about the most sweeping changes in Congress that we have seen in years, if not decades. Join your AAN colleagues at Neurology on the Hill from February 27-28, 2017, and help educate members of Congress so we can address critical health policy problems together. If selected, you will receive training to bring you up-to-date on key issues. Then, we will go to Capitol Hill for face-to-face meetings with congressional members and their staffs. The Academy will cover airfare expenses and hotel accommodations. There is a general registration fee of $\$ 150$, or $\$ 50$ for residents, fellows, and members residing in the Washington, DC, area. Encourage your colleagues to apply as well. Space is limited and fills quickly. Learn more and apply by November 20, 2016, at AAN.com/view/2017NOH. 


\section{Neurology}

\section{Rituximab in multiple sclerosis: A retrospective observational study on safety and efficacy \\ Jonatan Salzer, Rasmus Svenningsson, Peter Alping, et al.}

Neurology 2016;87;2074-2081 Published Online before print October 19, 2016

DOI 10.1212/WNL.0000000000003331

This information is current as of October 19, 2016

\section{Updated Information \&} Services

\section{Supplementary Material}

\section{References}

Citations

Subspecialty Collections

Permissions \& Licensing

Reprints including high resolution figures, can be found at: http://n.neurology.org/content/87/20/2074.full

Supplementary material can be found at: http://n.neurology.org/content/suppl/2016/10/19/WNL.0000000000003 331.DC1

http://n.neurology.org/content/suppl/2016/10/19/WNL.0000000000003 331.DC2

This article cites 19 articles, 6 of which you can access for free at: http://n.neurology.org/content/87/20/2074.full\#ref-list-1

This article has been cited by 2 HighWire-hosted articles: http://n.neurology.org/content/87/20/2074.full\#\#otherarticles

This article, along with others on similar topics, appears in the following collection(s):

\section{Class IV}

http://n.neurology.org/cgi/collection/class_iv Multiple sclerosis

http://n.neurology.org/cgi/collection/multiple_sclerosis

Information about reproducing this article in parts (figures,tables) or in its entirety can be found online at:

http://www.neurology.org/about/about_the_journal\#permissions

Information about ordering reprints can be found online:

http://n.neurology.org/subscribers/advertise

Neurology ${ }^{\circledR}$ is the official journal of the American Academy of Neurology. Published continuously since 1951, it is now a weekly with 48 issues per year. Copyright () 2016 American Academy of Neurology. All rights reserved. Print ISSN: 0028-3878. Online ISSN: 1526-632X.

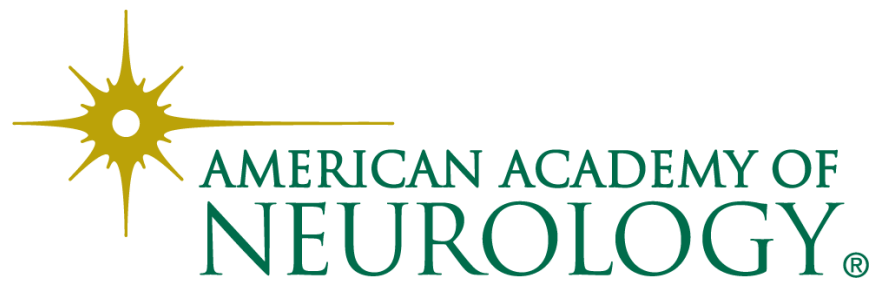

